Historic, archived document

Do not assume content reflects current scientific knowledge, policies, or practices. 



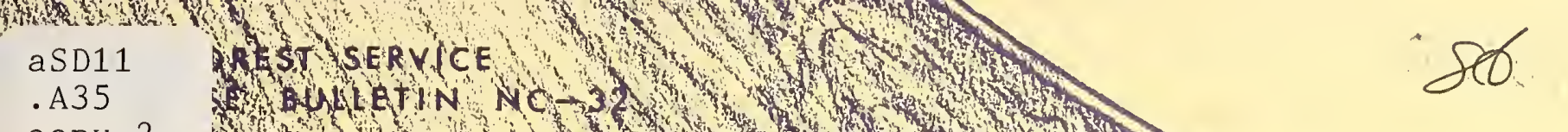

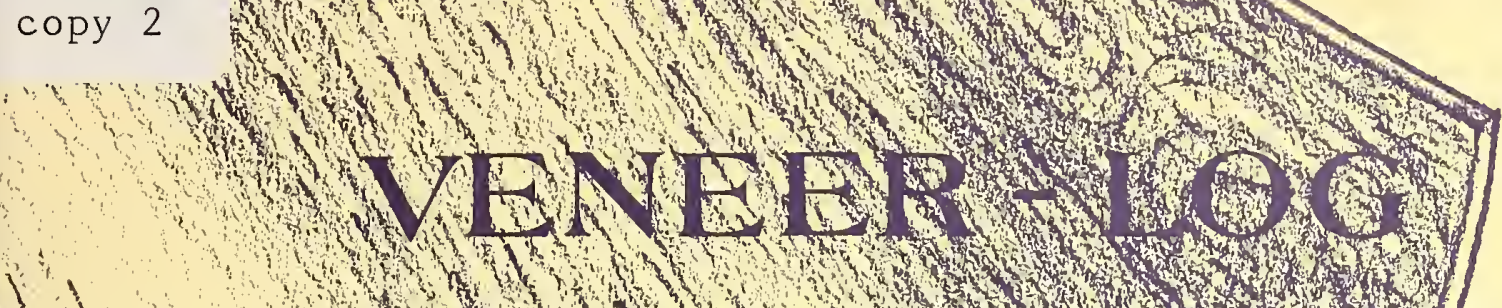

1 4,5
$m$

ANDWW W
RECHITS

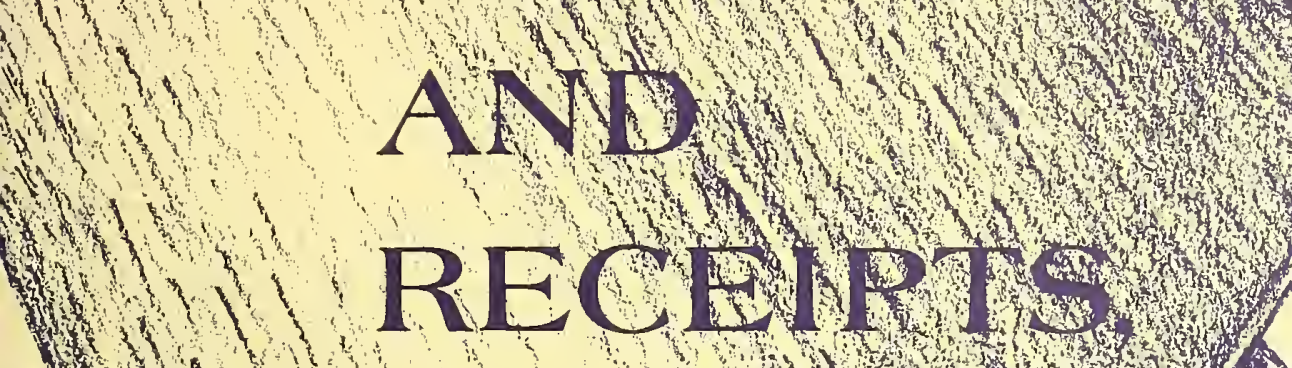

NORTH CENTR PREGION MIRTCBN?

1974

(n)

Janly, E, Blyth Jerohd Mahn

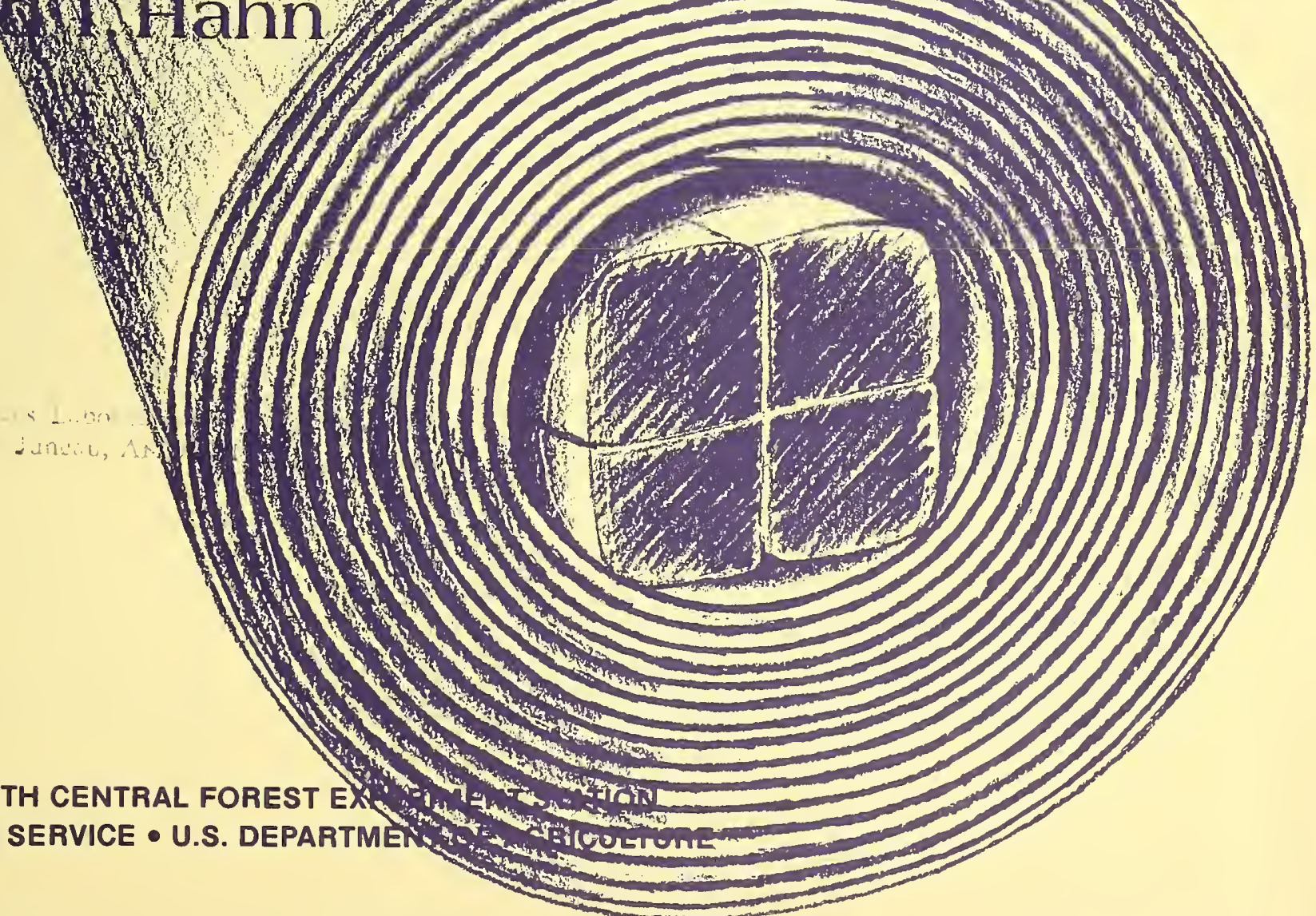

NORTH CENTRAL FOREST EX

FOREST SERVICE • U.S. DEPARTME 


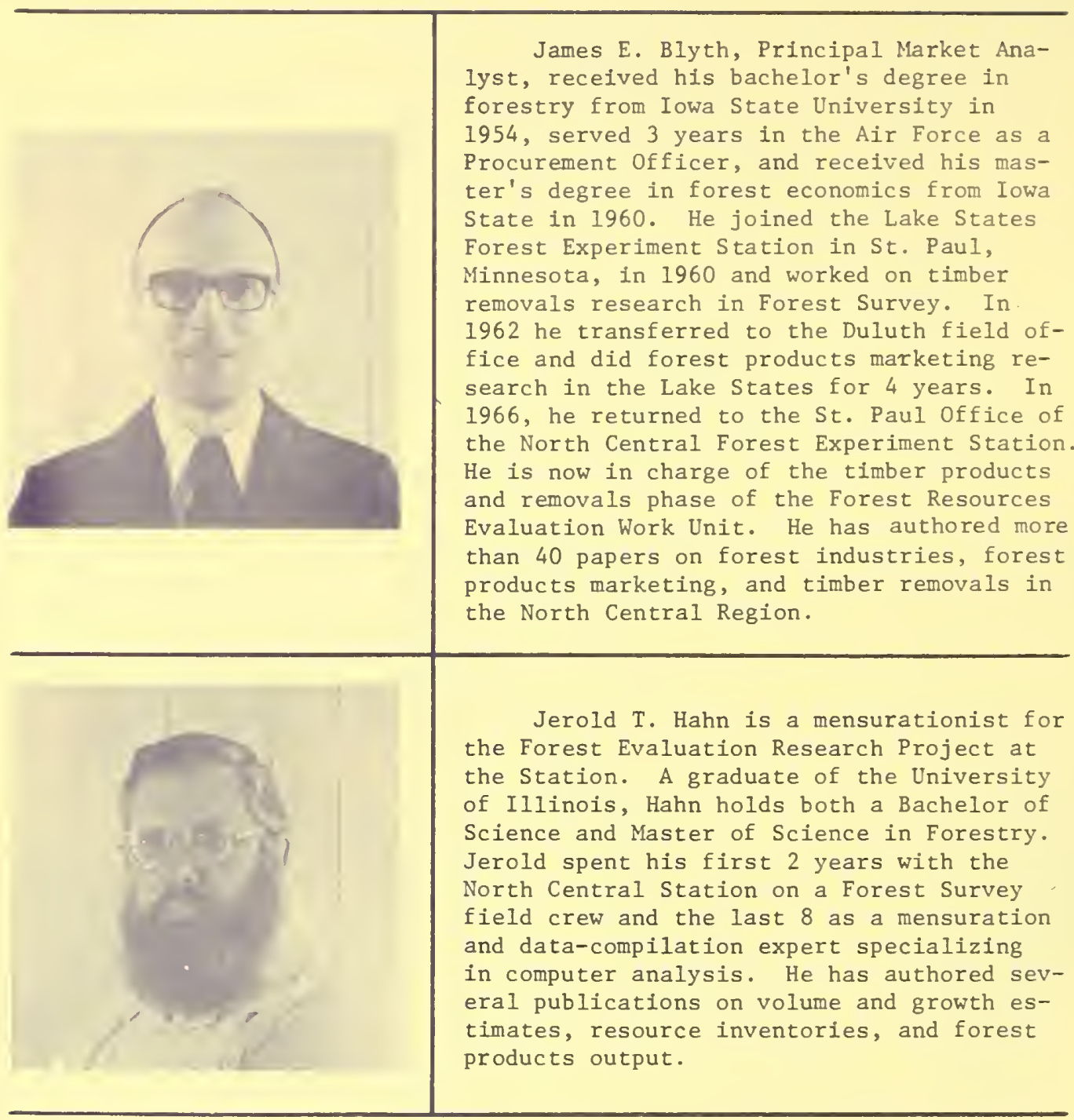

CONTENTS

Lake States... . . . . . . . . . . . . . 1

Central States . . . . . . . . . . . . 3

Appendix . . . . . . . . . . . . . . . 5

North Central Forest Experiment Station

John H. Ohman, Director

Forest Service - U.S. Department of Agriculture

Folwell Avenue

St. Paul, Minnesota 55108 


\title{
VENEER-LOG PRODUCTION AND RECEIPTS, \\ NORTH CENTRAL REGION, 1974
}

\author{
James E. Blyth and Jerold T. Hahn
}

This report of 1974 veneer-log production and recelpts ${ }^{1}$ in the North Central Region is divided into two sections--Lake States (Minnesota, Wisconsin, and Michigan) and Central States (Indiana, I1linois, Iowa, and Missouri)--because different species are grown and used as veneer logs in each area. Moreover, less information can be released about the Central States because more detailed data would reveal the operations of individual mills. Export data are reported for Canada but not for other countries.

Al1 but one veneer mill using North Central Region timber in 1974 reported their veneer-log receipts by species and State of origin. Their cooperation is gratefully acknowledged. Receipts were estimated for the nonresponding mill. Statistics for one large mill are not included in this report to avoid disclosure of its operations (fig. 1); data for the other large mill previously excluded are now included.

of the 58 active veneer mills in the Region in 1974 , 40 were standard ${ }^{2}$ veneer mills and 18 were container ${ }^{3}$ veneer mills. From 1972 to 1974 , one standard veneer mil1 and one container veneer mill closed; three standard veneer mills began operations.

\section{LAKE STATES}

\section{Production}

Lake States loggers harvested $68.7 \mathrm{mil}$ lion board feet of veneer logs in 1974.

${ }^{1}$ Production is the volume of veneer logs cut from a specific area; receipts are the volume of veneer logs received by mills in a specific area regardless of geographic source.

${ }^{2}$ Commercial, face, and specialty veneer mizls that manufacture veneer used in doorskins, fumiture, wall panels, specialty plywood, and similar items.

${ }^{3} \mathrm{Mi} Z Z_{s}$ that manufacture veneers used in boxes, crates, packing cases, and other wood veneer containers.
Five species comprised 84 percent of the total volume:

$\begin{array}{lr} & \begin{array}{c}\text { Thousand } \\ \text { board } \\ \text { feet }\end{array} \\ \text { Species } & 24.3 \\ \text { Aspen } & 11.7 \\ \text { Hard maple } & 11.1 \\ \text { Red oak } & 6.2 \\ \text { Elm } & 4.2\end{array}$

Most of the harvest (64.1 million board feet) was standard veneer logs; the remainder was container veneer logs.

Hard maple regained its position ahead of red oak as a veneer species (fig. 2). Excluding aspen, the major changes in veneer log production between 1972 and 1974 were:

$\begin{array}{lcc} & \begin{array}{c}\text { Thousand } \\ \text { board } \\ \text { feet }\end{array} & \text { Percent } \\ \text { Species } & & \\ \text { Hard maple } & +2,451 & +26 \\ \text { Red oak } & +1,404 & +14 \\ \text { Elm } & +1,061 & +21 \\ \text { Yellow birch } & -1,015 & -20 \\ \text { Soft maple } & +976 & +94\end{array}$

The long-term downtrend in the yellow birch harvest continued in 1974 .

Wisconsin produced 37.3 million board feet of logs. Aspen and red oak were the principal species cut. The elm harvest rose 1.3 million board feet from 1972, probably because Dutch Elm Disease was spreading rapidly through Wisconsin and many high quality logs were being salvaged from infected trees. Walnut and red oak were the primary species shipped outside the lake States from Wisconsin.

Michigan cut 25.9 million board feet of veneer logs, principally from aspen, hard maple, and yellow birch. The elm harvest dropped below one-half million board feet, 


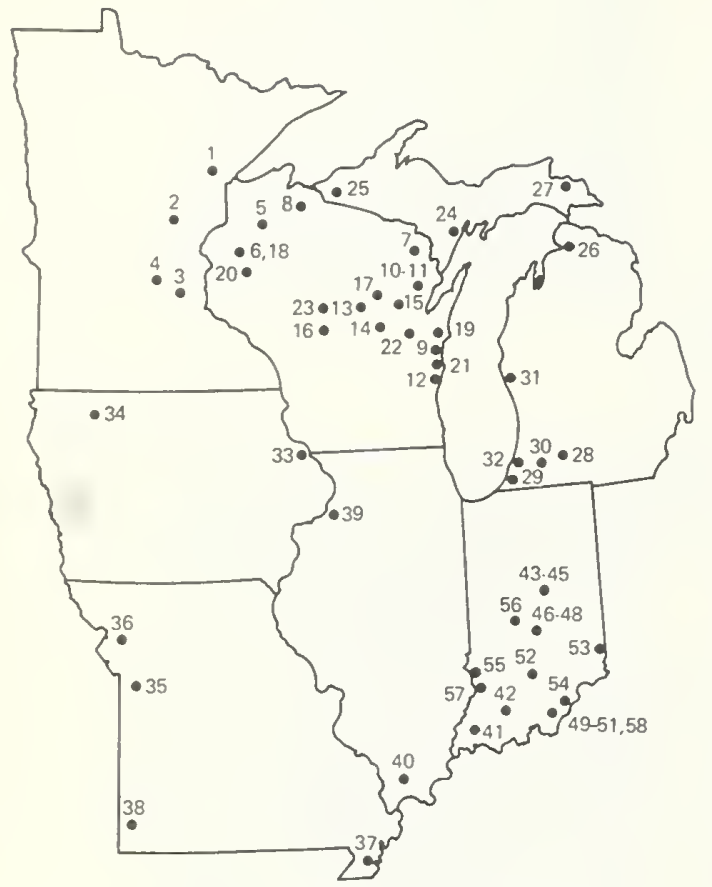

Figure 1.--Location of active veneer milzs in the North Central Region, 1974.
MANESOTA

\begin{tabular}{|c|c|c|c|}
\hline $\begin{array}{l}\text { Type of } \\
\text { m111 }\end{array}$ & $\begin{array}{l}\text { :Num- : } \\
: \text { ber: }\end{array}$ & Name of 0111 & Location \\
\hline Standard & $\begin{array}{l}1 \\
2\end{array}$ & $\begin{array}{l}\text { Dlamond International Co. } 1 \text { / } \\
\text { Wahkon Veneer M1lls }\end{array}$ & $\begin{array}{l}\text { Cloquet } \\
\text { Wahkon }\end{array}$ \\
\hline Contalner & $\begin{array}{l}3 \\
4\end{array}$ & $\begin{array}{l}\text { Allen hood Products, Inc. } \\
\text { Elk Rlver Box Co. }\end{array}$ & $\begin{array}{l}\text { Hopk1ns } \\
\text { Elk R1ver }\end{array}$ \\
\hline \multicolumn{4}{|c|}{ WISCONSIN } \\
\hline Standard & $\begin{array}{r}5 \\
6 \\
7 \\
8 \\
9 \\
10 \\
11 \\
12 \\
13 \\
14 \\
15 \\
16 \\
17\end{array}$ & $\begin{array}{l}\text { Birchwood Lumber \& Veneer Co. } \\
\text { B1rchwood } \mathrm{Mfg} \text {. Co. } \\
\text { Loulsiana-Pacif1c Corp. } \\
\text { Loulsiana-Pacif1c Corp. } \\
\text { Eggers Plywood Co. } \\
\text { G1llett Veneer \& Plywood Co. } \\
\text { L1nwood, Inc. } \\
\text { Larson Plywood Co., Inc. } \\
\text { Hatley Veneer Co. Inc. } \\
\text { Marion Plywood Corp. } \\
\text { Weber Veneer \& Plywood Co. } \\
\text { Weyerhaeuser Co. } \\
\text { Wisconsin Timber \& Land Co. }\end{array}$ & $\begin{array}{l}\text { B1rchwood } \\
\text { Rice Lake } \\
\text { Coodman } \\
\text { Mellen } \\
\text { Two Rivers } \\
\text { G1llett } \\
\text { G1llett } \\
\text { Sheboygan } \\
\text { Matley } \\
\text { Marion } \\
\text { Shawano } \\
\text { Marshfield } \\
\text { Matoon }\end{array}$ \\
\hline Containet & $\begin{array}{l}18 \\
19 \\
20 \\
21 \\
22 \\
23\end{array}$ & $\begin{array}{l}\text { Brunette Box } \& \text { Veneer } \\
\text { Dufeck Mfg. Co. } \\
\text { Ebner Box Factory } \\
\text { Konz Cantalner Co. } \\
\text { Seymour Woodenware Co. } \\
\text { Standard Container Co. }\end{array}$ & $\begin{array}{l}\text { Rlce Lake } \\
\text { Denmark } \\
\text { Cameron } \\
\text { Howard Grove } \\
\text { Seymour } \\
\text { Edgar }\end{array}$ \\
\hline \multicolumn{4}{|c|}{ MICHIGAN } \\
\hline Standard & $\begin{array}{l}24 \\
25 \\
26 \\
27\end{array}$ & $\begin{array}{l}\text { Anthony \& Co. } \\
\text { Iron Wood Products } \\
\text { Manthe1 Bros. } \\
\text { Soo Hardwoods, Inc. }\end{array}$ & $\begin{array}{l}\text { Escanaba } \\
\text { Bessemer } \\
\text { Petoskey } \\
\text { Sault Ste. Mar1e }\end{array}$ \\
\hline Container & $\begin{array}{l}28 \\
29 \\
30 \\
31 \\
32\end{array}$ & $\begin{array}{l}\text { A. C. Hensel Mfg. Co. } \\
\text { Nelson R. Anderson Co. } \\
\text { Frult Growers Package Co. } \\
\text { Mich1gan Crate \& Basket } \\
\text { R1vergide Package Co. }\end{array}$ & $\begin{array}{l}\text { Augusta } \\
\text { Stevensville } \\
\text { Paw Paw } \\
\text { Shelby } \\
\text { Riverside }\end{array}$ \\
\hline \multicolumn{4}{|c|}{ IOWA } \\
\hline Standard & $\begin{array}{l}33 \\
34\end{array}$ & $\begin{array}{l}\text { R. S. Bacon Veneer Co. } \\
\text { Iowa Veneers, Inc. }\end{array}$ & $\begin{array}{l}\text { Dubuque } \\
\text { Spencer }\end{array}$ \\
\hline \multicolumn{4}{|c|}{ MISSOURI } \\
\hline \multirow{2}{*}{$\begin{array}{l}\text { Standard } \\
\text { Container }\end{array}$} & 35 & $\begin{array}{l}\text { Var1o Veneer Corp. } \\
\text { M1saour } 1 \text { Valley Walnut Co. }\end{array}$ & $\begin{array}{l}\text { Pleasant H1ll } \\
\text { St. Joseph }\end{array}$ \\
\hline & $\begin{array}{l}37 \\
38\end{array}$ & $\begin{array}{l}\text { Betz T1pton Veneer Co. } \\
\text { Crumbl18s Wood Products }\end{array}$ & $\begin{array}{l}\text { Caruthersville } \\
\text { Neosho }\end{array}$ \\
\hline \multicolumn{4}{|c|}{ ILIINOIS } \\
\hline Standard & 39 & Swords Veneer \& Lumber $\mathrm{Co}$. & Rock Island \\
\hline Container & 40 & $\begin{array}{l}\text { The Martin Bros. Contalner } \\
\text { \& TImber Products, Inc. }\end{array}$ & Karnak \\
\hline \multicolumn{4}{|c|}{ INDIANA } \\
\hline Standard & $\begin{array}{l}41 \\
42 \\
43 \\
44 \\
45 \\
46 \\
47 \\
48 \\
49 \\
50 \\
51 \\
52 \\
53 \\
54 \\
55 \\
56\end{array}$ & $\begin{array}{l}\text { Evangville Veneer Co. } \\
\text { Jasper Veneer M1lls } \\
\text { Central Veneer, Inc. } \\
\text { Curry Miller Veneers, Inc. } \\
\text { Indiana Veneers, Inc. } \\
\text { Dav1d R. Webb Co. } \\
\text { Amos Thompoon Corp. } \\
\text { H1ll Bros. Veneer Co., Inc. } \\
\text { B. L. Curry \& Sons } \\
\text { Chester B. Stem, Inc. } \\
\text { Adama Custom Veneer Co. } \\
\text { National Veneer \& Lumber Co. } \\
\text { Plerson-Holowell Co., Inc. } \\
\text { Roberts \& Strack Veneer Co. } \\
\text { Ford Sawmill Inc. (Veneer Div.) } \\
\text { The1sing Veneer Co., Inc. }\end{array}$ & $\begin{array}{l}\text { Evangv1lle } \\
\text { Jasper } \\
\text { Ind1anapol1s } \\
\text { Ind1anapol1s } \\
\text { Ind1anapol1s } \\
\text { Ed1nburg } \\
\text { Edinburg } \\
\text { Ed1nburg } \\
\text { New Albany } \\
\text { New Albany } \\
\text { New Albany } \\
\text { Seymour } \\
\text { Lawrenceburg } \\
\text { Clarksille } \\
\text { Vincennes } \\
\text { Mooregv1lle }\end{array}$ \\
\hline Container & $\begin{array}{l}57 \\
58\end{array}$ & $\begin{array}{l}\text { Farrell Box Co. Inc. } \\
\text { New Albany Box \& Basket Co., Inc. }\end{array}$ & $\begin{array}{l}\text { Decker } \\
\text { New Albany }\end{array}$ \\
\hline
\end{tabular}

1 Recelpta not recorded to avold disclosure of operations at th1s m111. 


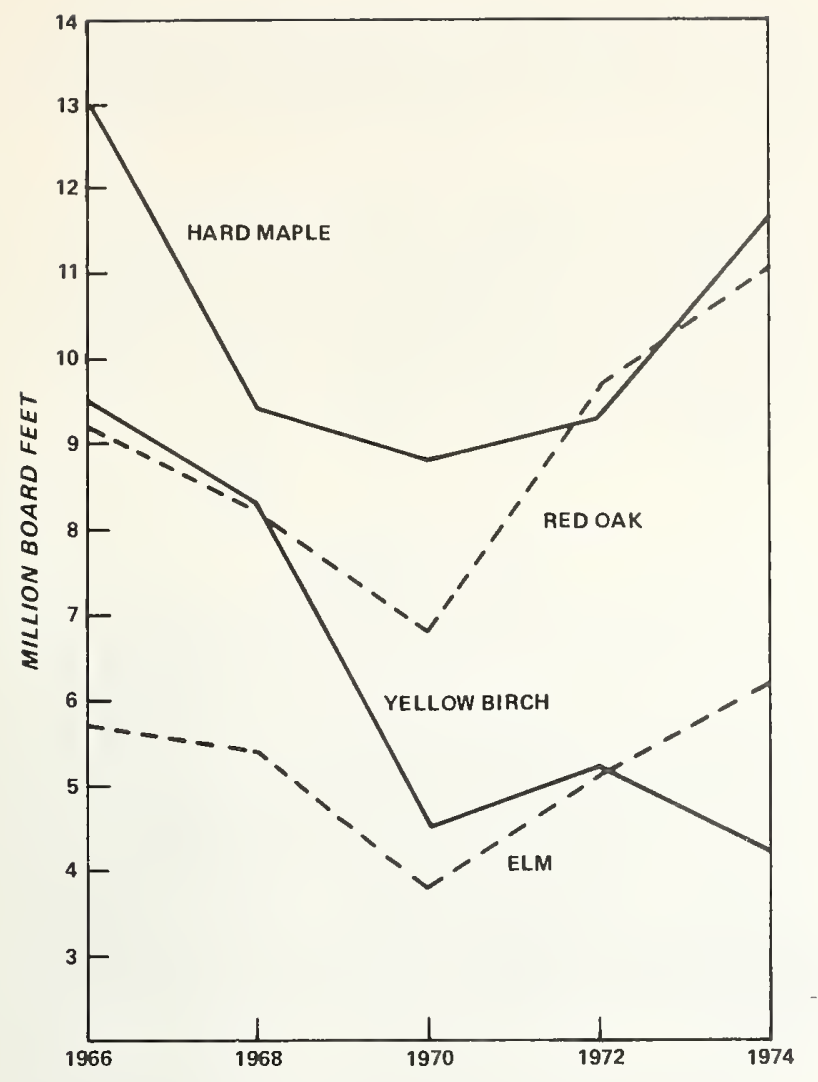

Figure 2.--Veneer-log production of four major species for selected years, Lake States, 1966-1974.

probably because the quantity of elm remaining in Michigan is small. Of the total cut, 39 percent was shipped to Wisconsin and 12 percent was shipped beyond the Lake States. Important species shipped out-of-State were hard maple, yellow birch, and red oak.

In Minnesota, 93 percent of the 5.4 million board feet of veneer logs cut was procured by out-of-State mills. Cottonwood, aspen, elm, and red oak were the dominant species cut.

\section{Mi11 Receipts}

Lake States veneer mills recelved 67.5 million board feet of logs in 1974 including imports of 4.1 million board feet. Imports from Canada fell 1 million board feet ( 33 percent) compared to 1972 while log receipts from other States (primarily Iowa) rose half a million board feet. Excluding aspen, major increases in demand over 1972 were for hard maple, soft maple, and red oak.

Nineteen Wisconsin veneer mills received $41.6 \mathrm{million}$ board feet of logs, 5.1 million board feet more than in 1972. Red oak, hard maple, and elm were the primary species procured. One-fourth of the volume came from Michigan including more than half of the hard maple and yellow birch receipts.

Michigan's mills procured 25.3 million board feet of veneer logs. Imports from Canada were off 44 percent from 1972 and no other wood was purchased outside the Lake States.

Minnesota veneer plants received 0.6 million board feet of logs, up 17 percent from 1972 .

\section{Plant Residue}

More than 99 percent of the coarse residue generated at Lake States veneer mills in 1974 was used. On the other hand, 36 percent of the fine residue and 41 percent of the bark were not used. The primary use (69 percent) for coarse residue was in fiber products. Important uses for fine residue were industrial fuel (32 percent) and fiber products (28 percent). More than half (58 percent) the bark was used for industrial fuel. Disposal problems appear to be significant for fine residue and bark. The only problem with coarse residue disposal might be in finding higher value markets.

\section{CENTRAL STATES}

\section{Production}

Central States veneer-log production continued to rebound in 1974 from the low point in 1970 (fig. 3). The harvest was 29.1 million board feet, up 11 percent from 1972. Eighty-five percent (24.8 million board feet) was standard veneer logs, the remainder was used for container veneer.

${ }^{4}$ Plant residue at veneer mills is classed as: (1) bark; (2) coarse (wood)-suitable for chipping, such as veneer cores; or (3) fine (wood)--not suitable for chipping, such as veneer clippings. 


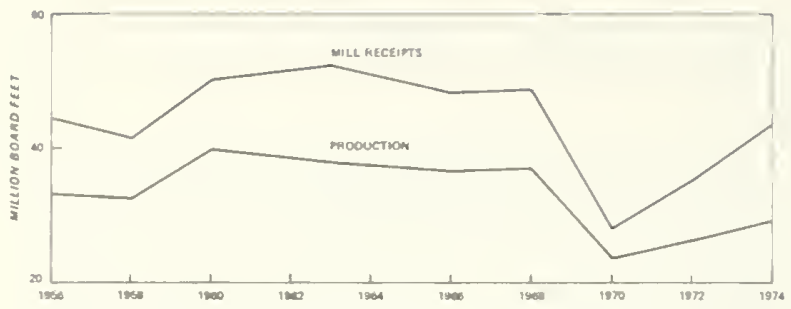

Figure 3.--Veneer-log production and mill receipts in the Central States, 19561974.

Principal species cut were:

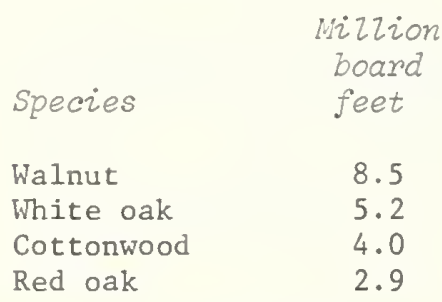

Walnut and the oaks were used primarily in furniture and decorative paneling. Cottonwood was used chiefly for containers and pallets.

Important harvest changes between 1972 and 1974 were:

$\begin{array}{llc} & \begin{array}{c}\text { Thousand } \\ \text { board } \\ \text { feet }\end{array} & \text { Percent } \\ \text { Species } & & +56 \\ \text { White oak } & +1,861 & +70 \\ \text { Red oak } & +1,217 & -15 \\ \text { Walnut } & -1,486 & -51 \\ \text { Pecan (hickory) } & -1,232 & \end{array}$

Red oak and white oak harvests were the highest recorded in the last 2 decades.
Exports moved slightly higher ( 3 percent) than in 1972 to 5.5 million board feet; they were mainly to Kentucky (46 percent), Wisconsin (34 percent), and Ohio (13 percent).

Indiana loggers cut half of the Central States veneer $\log$ volume. Iowa edged out Illinois for second place in production after being 1.5 million board feet behind in 1972. Only Missouri cut a smaller volume of veneer logs than in 1972.

\section{Mill Receipts}

Twenty-six Central States mills received 39.7 million board feet of veneer logs in 1974 , a rise of 4.7 million board feet from 1972. These mills imported 16.2 million board feet of logs from 20 other States and Canada including more than 1 million board feet from each of 6 States-Ohio, Kentucky, Oklahoma, Wisconsin, Michigan, and Pennsylvania. Major imports included black cherry from Pennsylvania, pecan from Oklahoma, and white oak from Ohio.

Indiana's 18 veneer mills procured 76 percent of the total $\log$ receipts in 1974 including 85 percent or more of the black cherry, hard maple, pecan, red oak, white oak, and yellow-poplar.

\section{Plant Residue}

Most wood residue (90 percent) was used so the only significant disposal problem may be in upgrading the uses. On the other hand, 30 percent of the bark was disposed of as waste, a significant increase from only 12 percent in 1972. Industrial fuel was the primary use for both wood and bark residue. 


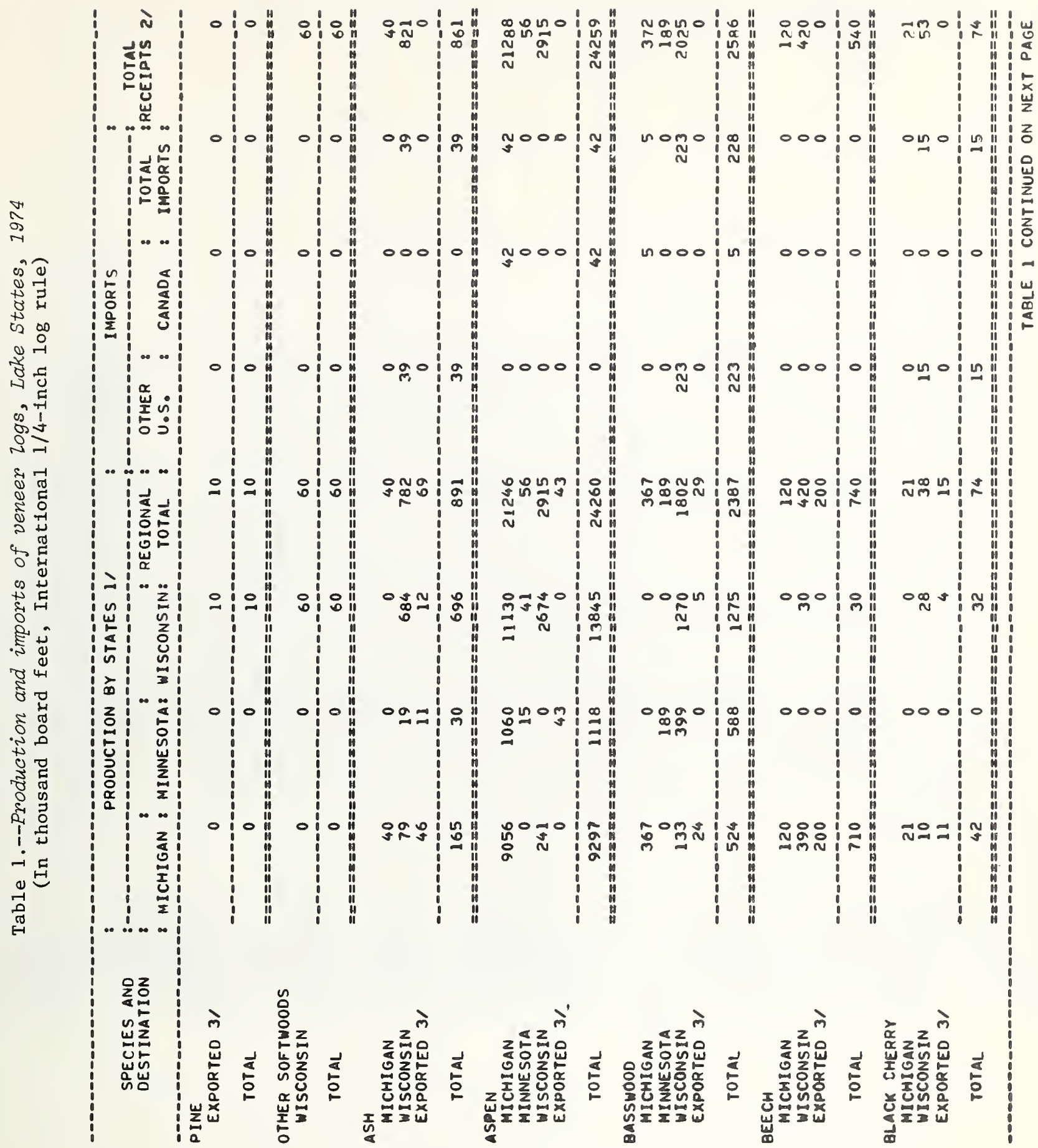




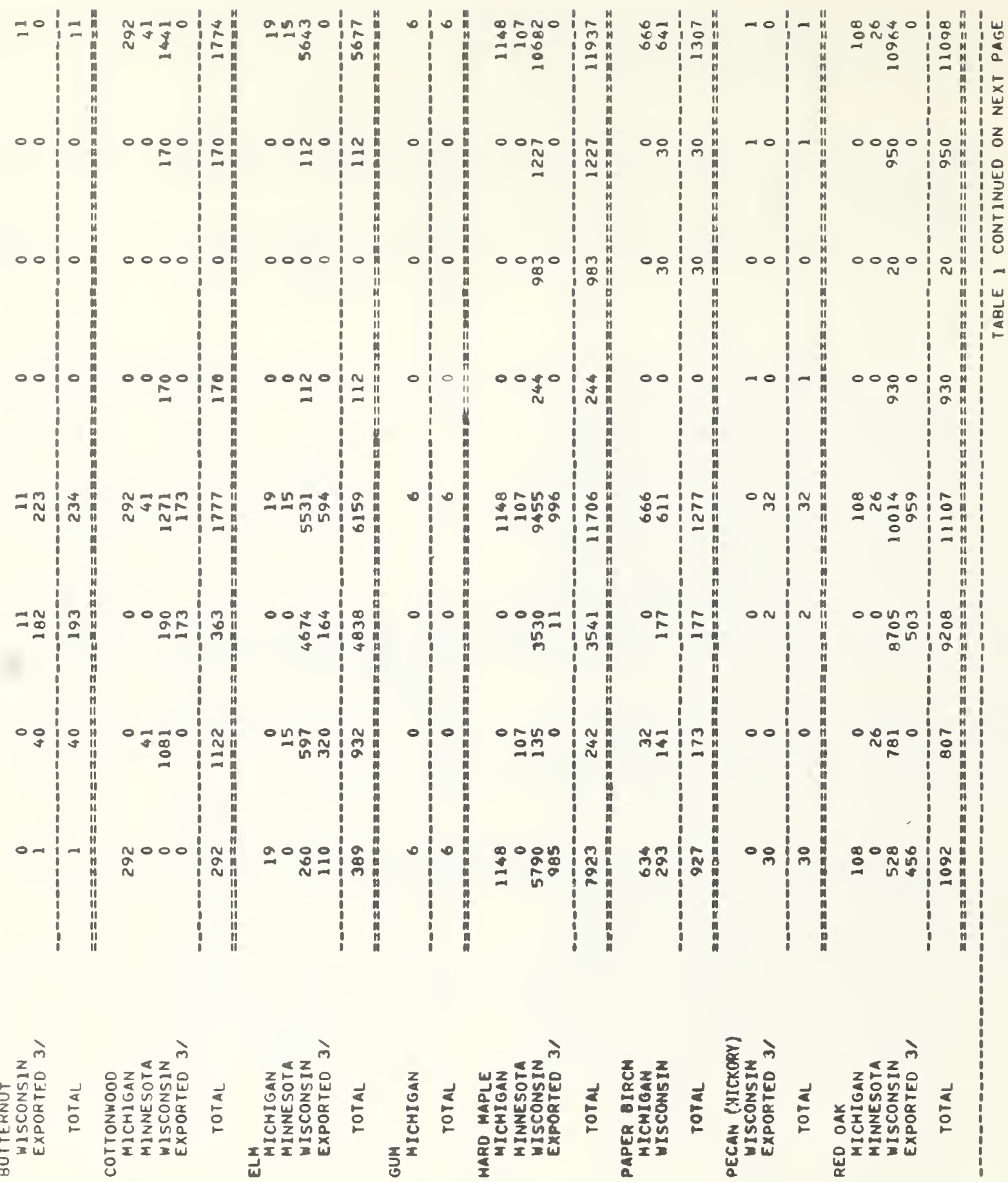




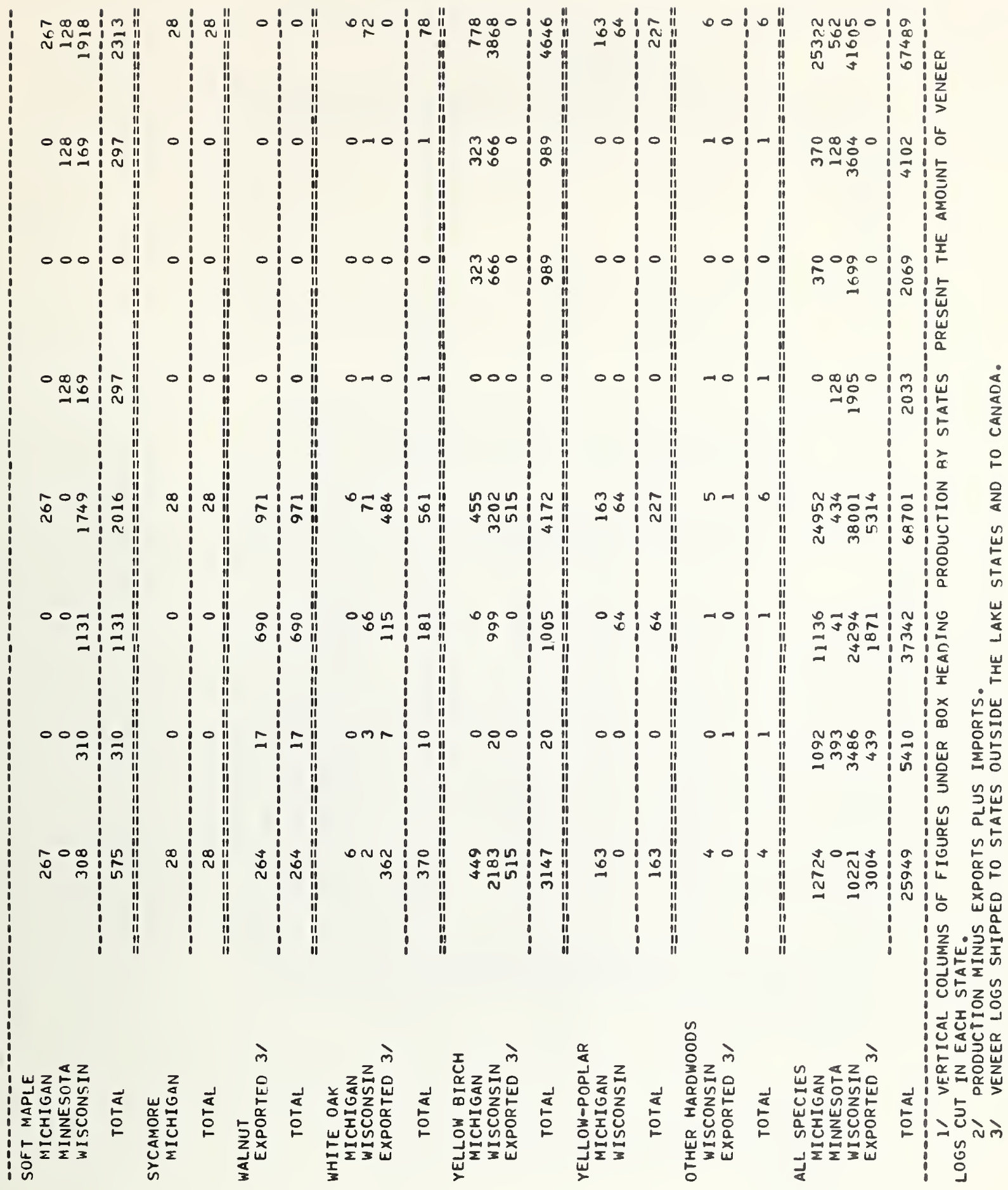


Table 2.--Milzs using five species of Lake States veneer logs, by location, 1974

(In numbers)

\begin{tabular}{|lrrrrr|}
\hline \multicolumn{2}{|c|}{ Location } & : Red oak : Hard maple: Yellow birch: Elm: & Aspen \\
\hline Lake States & 15 & 17 & 11 & 17 & 16 \\
Indiana & 5 & 7 & -- & 2 & -- \\
Illinols & 1 & -- & -- & 1 & -- \\
Iowa & 2 & 1 & -- & 2 & - \\
Ohio & -- & 2 & -- & -- & -- \\
Kentucky & 1 & 1 & -- & 1 & - \\
Canada & 1 & 1 & 1 & 1 & 1 \\
\cline { 2 - 7 } Total & 25 & 29 & 12 & 24 & 17 \\
\hline
\end{tabular}

Table 3.--Veneer-log production and receipts in the Lake States by species, 1972 and 1974

(In thousand board feet, International 1/4-1nch log rule)

\begin{tabular}{|c|c|c|c|c|c|c|}
\hline Species & \multicolumn{3}{|c|}{ Production } & \multicolumn{3}{|c|}{ Receipts } \\
\hline & 1972 & 1974 & : change & $\therefore 1972$ & : 1974 & : change \\
\hline $\begin{array}{l}\text { Ash } \\
\text { Aspen }\end{array}$ & 603 & 891 & +288 & 629 & 861 & +232 \\
\hline $\begin{array}{l}\text { Aspen } \\
\text { Basswood }\end{array}$ & $\frac{1 /}{72}$ & $1 /$ & $1 /$ & $1 /$ & $1 /$ & $1 /$ \\
\hline $\begin{array}{l}\text { Basswood } \\
\text { Beech }\end{array}$ & 2873 & 2387 & -486 & 3100 & 2586 & -514 \\
\hline $\begin{array}{l}\text { Beech } \\
\text { Black cherry }\end{array}$ & $\begin{array}{r}1065 \\
151\end{array}$ & 740 & -325 & 748 & 540 & -208 \\
\hline Butternut & $\begin{array}{l}151 \\
346\end{array}$ & $\begin{array}{r}74 \\
234\end{array}$ & $\begin{array}{r}-77 \\
-112\end{array}$ & $\begin{array}{r}128 \\
2\end{array}$ & $\begin{array}{l}74 \\
11\end{array}$ & $\begin{array}{r}-54 \\
+9\end{array}$ \\
\hline Cottonwood & 1268 & 1777 & +509 & 1276 & $\begin{array}{r}11 \\
1774\end{array}$ & $\begin{array}{r}+9 \\
+498\end{array}$ \\
\hline Elm & 5098 & 6159 & +1061 & 5255 & 5677 & +422 \\
\hline Hard maple & 9255 & 11706 & +2451 & 9421 & 11937 & +2516 \\
\hline Paper birch & 1223 & 1277 & +54 & 1457 & 1307 & -150 \\
\hline Pecan (hickory) & 86 & 32 & -54 & 4 & 1 & -3 \\
\hline Red oak & 9703 & 11107 & +1404 & 10089 & 11098 & +1009 \\
\hline Soft maple & 1040 & 2016 & +976 & 1185 & 2313 & +1128 \\
\hline Walnut & 1376 & 971 & -405 & -- & -- & -- \\
\hline White oak & 287 & 561 & +274 & 202 & 78 & -124 \\
\hline Yellow birch & 5187 & 4172 & -1015 & 6349 & 4646 & -1703 \\
\hline Other species & 57 & 337 & +280 & 49 & 327 & +278 \\
\hline Total & 39618 & 44441 & +4823 & 39894 & 43230 & +3336 \\
\hline
\end{tabular}

$1 /$ Comparison was not meaninfgul because log production and receipts for one veneer mill were included in 1974 but not in previous years. 


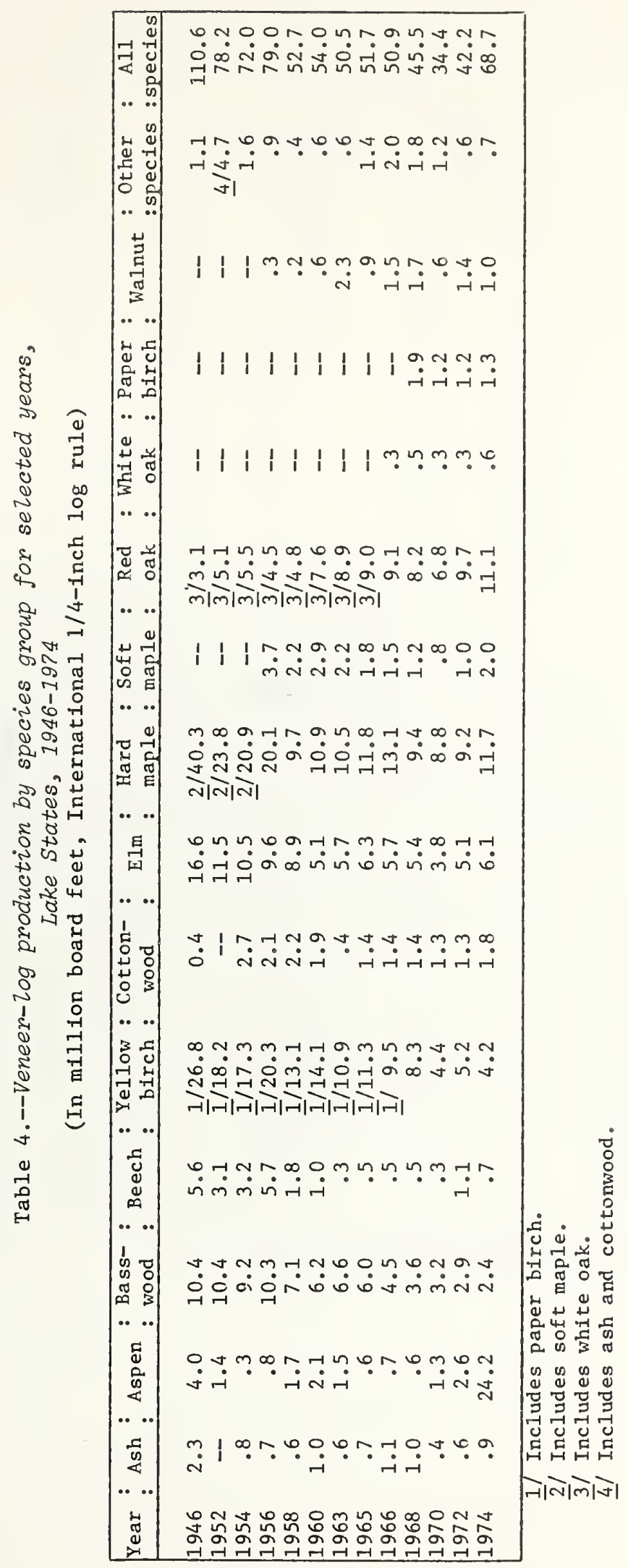


Table 5.--Veneer-log production for selected years and type of industry, Lake states, $1946-1974$

(In million board feet, International $1 / 4$-inch 10g.rule)

\begin{tabular}{|cccr|}
\hline Year & : & Standard & $\vdots$ \\
\hline & Contalner & : & Total \\
\hline 1946 & 72.7 & 37.9 & 110.6 \\
1948 & 71.7 & 45.1 & 116.8 \\
1950 & 47.3 & 39.3 & 86.6 \\
1952 & 46.8 & 31.4 & 78.2 \\
1954 & 45.3 & 26.7 & 72.0 \\
1956 & 51.6 & 27.4 & 79.0 \\
1958 & 40.3 & 12.4 & 52.7 \\
1960 & 46.5 & 7.5 & 54.0 \\
1962 & 41.0 & 6.6 & 47.6 \\
1963 & 43.9 & 6.6 & 50.5 \\
1965 & 44.6 & 7.1 & 51.7 \\
1966 & 45.0 & 5.9 & 50.9 \\
1968 & 40.6 & 4.9 & 45.5 \\
1970 & 29.4 & 5.0 & 34.4 \\
1972 & 38.0 & 4.2 & 42.2 \\
1974 & 64.1 & 4.6 & 68.7 \\
\hline
\end{tabular}

Table 6.--Lake States veneer-log production, receipts, exports, and imports for selected years, 1946-1972

(In million board feet, International 1/4-inch log rule)

\begin{tabular}{|c|c|c|c|c|c|}
\hline Year & $\begin{array}{l}\text { : Produced In the Lake: } \\
\text { : States and recelved : } \\
\text { : at Lake States Mills: }\end{array}$ & Exported $1 /$ & $:$ Imported $2 /$ & $\begin{array}{l}: \text { Total : } \\
\text { :production In: } \\
: \text { Lake States : }\end{array}$ & $\begin{array}{l}\text { Total } \\
\text { receipts in } \\
\text { Lake States }\end{array}$ \\
\hline 1946 & 109.7 & 0.9 & 5.9 & 110.6 & 115.6 \\
\hline 1948 & 116.0 & .8 & 12.0 & 116.8 & 128.0 \\
\hline 1950 & 86.0 & .6 & 15.7 & 86.6 & 101.7 \\
\hline 1952 & 77.8 & .4 & 12.1 & 78.2 & 89.9 \\
\hline 1954 & 71.6 & .4 & 12.1 & 72.0 & 83.7 \\
\hline 1956 & 78.7 & .3 & 13.4 & 79.0 & 92.1 \\
\hline 1958 & 51.5 & 1.2 & 6.0 & 52.7 & 57.5 \\
\hline 1960 & 51.9 & 2.1 & 12.5 & 54.0 & 64.4 \\
\hline 1963 & 45.0 & 5.5 & 7.7 & 50.5 & 52.7 \\
\hline 1965 & 49.0 & 2.7 & 7.6 & 51.7 & 56.6 \\
\hline 1966 & 45.8 & 5.1 & 9.6 & 50.9 & 55.4 \\
\hline 1968 & 39.7 & 5.8 & 7.9 & 45.5 & 47.6 \\
\hline 1970 & 31.4 & 3.0 & 5.6 & 34.4 & 37.0 \\
\hline 1972 & 37.7 & 4.5 & 4.6 & 42.2 & 42.3 \\
\hline 1974 & 63.4 & 5.3 & 4.1 & 68.7 & 67.5 \\
\hline
\end{tabular}

1/ From Lakes States to other States and Canada.

2/ From other States and Canada Into Lakes States. 
Table 7.--Veneer-log receipts in the Lake States by species groups for selected years, 1952-1974

\begin{tabular}{|c|c|c|c|c|c|c|c|c|c|c|c|c|c|c|c|c|c|}
\hline Tear & : Ash: & Aspen & $\begin{array}{l}\text { : Bas8- } \\
: \text { wood }\end{array}$ & Beech & $\begin{array}{l}\text { : Yellow } \\
: \text { birch }\end{array}$ & $:$ & $\begin{array}{l}\text { Cotton-: } \\
\text { wood: }\end{array}$ & Elm & $\begin{array}{l}\text { : Hard } \\
\text { : maple }\end{array}$ & $\begin{array}{l}: \text { Soft } \\
: \text { maple }\end{array}$ & $:$ & $\begin{array}{l}\text { Red } \\
\text { oak }\end{array}$ & $:$ & $\begin{array}{l}\text { White } \\
\text { oak }\end{array}$ & $\begin{array}{l}\text { : Paper } \\
: \text { birch }\end{array}$ & $\begin{array}{l}\text { : Other : } \\
: \text { specles: }\end{array}$ & $\begin{array}{c}\text { A11 } \\
\text { spec1es }\end{array}$ \\
\hline 1952 & - & 1.4 & 10.9 & 3.2 & $1 / 27.1$ & & - & 11.7 & $2 / 24.9$ & - & & $3 / 5.3$ & & - & - & $4 / 5.4$ & 89.9 \\
\hline 1954 & .8 & .3 & 10.3 & 3.2 & $\bar{I} / 25.0$ & & 2.7 & 10.9 & $\overline{2} / 22.4$ & -- & & $3 / 6.5$ & & - & - & 1.6 & 83.7 \\
\hline 1956 & .7 & .8 & 10.6 & 5.7 & $\overline{1} / 28.5$ & & 3.0 & 10.2 & 21.9 & 4.7 & & $\frac{3}{3} / 5.0$ & & - & - & 1.0 & 92.1 \\
\hline 1958 & .6 & 1.7 & 7.3 & 1.8 & $\overline{1} / 17.2$ & & 2.2 & 9.2 & 9.6 & 2.5 & & $\overline{3} / 5.0$ & & - & - & .4 & 57.5 \\
\hline 1960 & 1.1 & 2.1 & 6.8 & .9 & $\overline{1} / 18.9$ & & 3.3 & 6.2 & 12.2 & 3.6 & & $\overline{3} / 8.4$ & & - & - & .9 & 64.4 \\
\hline 1963 & .7 & 1.6 & 6.7 & .3 & $1 / 14.3$ & & .7 & 4.9 & 11.1 & 2.6 & & $\overline{3} / 9.3$ & & - & -- & .5 & 52.7 \\
\hline 1965 & .7 & .6 & 6.3 & .5 & $\bar{I} / 14.5$ & & 2.1 & 6.3 & 13.2 & 1.9 & & $\underline{\overline{3}} / 9.3$ & & - & - & 1.2 & 56.6 \\
\hline 1966 & 1.1 & .7 & 4.8 & .5 & $\overline{1} / 14.7$ & & 2.3 & 5.8 & 12.8 & 1.8 & & 9.7 & & .2 & - & 1.0 & 55.4 \\
\hline 1968 & 1.0 & .6 & 3.9 & .5 & 10.2 & & 2.1 & 5.2 & 9.0 & 1.3 & & 8.3 & & .2 & 4.0 & 1.3 & 47.6 \\
\hline 1970 & .3 & 1.3 & 3.6 & .3 & 6.1 & & 1.4 & 3.8 & 9.0 & .9 & & 7.7 & & .2 & 1.9 & .5 & 37.0 \\
\hline 1972 & .6 & 2.4 & 3.1 & .7 & 6.3 & & 1.3 & 5.3 & 9.4 & 1.2 & & 10.1 & & .2 & 1.5 & .2 & 42.3 \\
\hline 1974 & .9 & 24.3 & 2.6 & .5 & 4.6 & & 1.8 & 5.7 & 11.9 & 2.3 & & 11.1 & & .1 & 1.3 & .4 & 67.5 \\
\hline
\end{tabular}

1/ Includes paper birch.

2/ Includes soft maple.

3/ Includes white oak.

$\frac{1}{4} /$ Includes ash and cottonwood.

Table 8.--Production and disposition of hardwood veneer plant residue by type of residue and use, Lake States, 1974

(In thousand cubic feet)

\begin{tabular}{|c|c|c|c|c|}
\hline State and & $:$ & Wood residue & & Bark \\
\hline type of use & Total & :Coarse 1/: & Fine 2/ & Bark. \\
\hline Michigan & & & & \\
\hline Fiber products & 625.6 & 625.6 & -- & -- \\
\hline Industrial fuel & 166.6 & 23.2 & 143.4 & 144.1 \\
\hline Domestic fuel & 16.0 & 16.0 & -- & -- \\
\hline Miscellaneous $3 /$ & 38.9 & 38.9 & -- & 16.8 \\
\hline Not used 4 I & 1001.6 & 5.4 & 996.2 & 624.0 \\
\hline Total - & 1848.7 & 709.1 & 1139.6 & 784.9 \\
\hline Minnesota & & & & \\
\hline Industrial fuel & 5.1 & 2.4 & 2.7 & 3.4 \\
\hline Domestic fuel & 10.3 & 9.8 & .5 & 8.0 \\
\hline Miscellaneous 3/ & 8.0 & 3.6 & 4.4 & -- \\
\hline Not used 4/ & 17.7 & -- & 17.7 & 6.1 \\
\hline Total & 41.1 & 15.8 & 25.3 & 17.5 \\
\hline Wisconsin & & & & \\
\hline Fiber products & 1531.3 & 685.2 & 846.1 & -- \\
\hline Industrial fuel & 1017.7 & 190.3 & 827.4 & 1060.3 \\
\hline Domestic fuel & 23.5 & 23.5 & -- & -- \\
\hline Miscellaneous $3 /$ & 385.5 & 256.1 & 129.4 & 4.5 \\
\hline Not used 4 / & 74.8 & 8.4 & 66.4 & 223.1 \\
\hline Total & 3032.8 & 1163.5 & 1869.3 & 1287.9 \\
\hline Al1 Lake States & & & & \\
\hline Fiber products & 2156.9 & 1310.8 & 846.1 & -- \\
\hline Industrial fuel & 1189.4 & 215.9 & 973.5 & 1207.8 \\
\hline Domestic fuel & 49.8 & 49.3 & .5 & 8.0 \\
\hline Miscellaneous $3 /$ & 432.4 & 298.6 & 133.8 & 21.3 \\
\hline Not used $\underline{4}$ & 1094.1 & 13.8 & 1080.3 & 853.2 \\
\hline Total & 4922.6 & 1888.4 & 3034.2 & 2090.3 \\
\hline
\end{tabular}

1/ Suitable for chipping such as veneer cores.

2/ Not suitable for chippine such as veneer clippings. 1tems.

3/ Livestock bedding, mulch, small dimension and specialty

4/ Including residues burned as waste. 


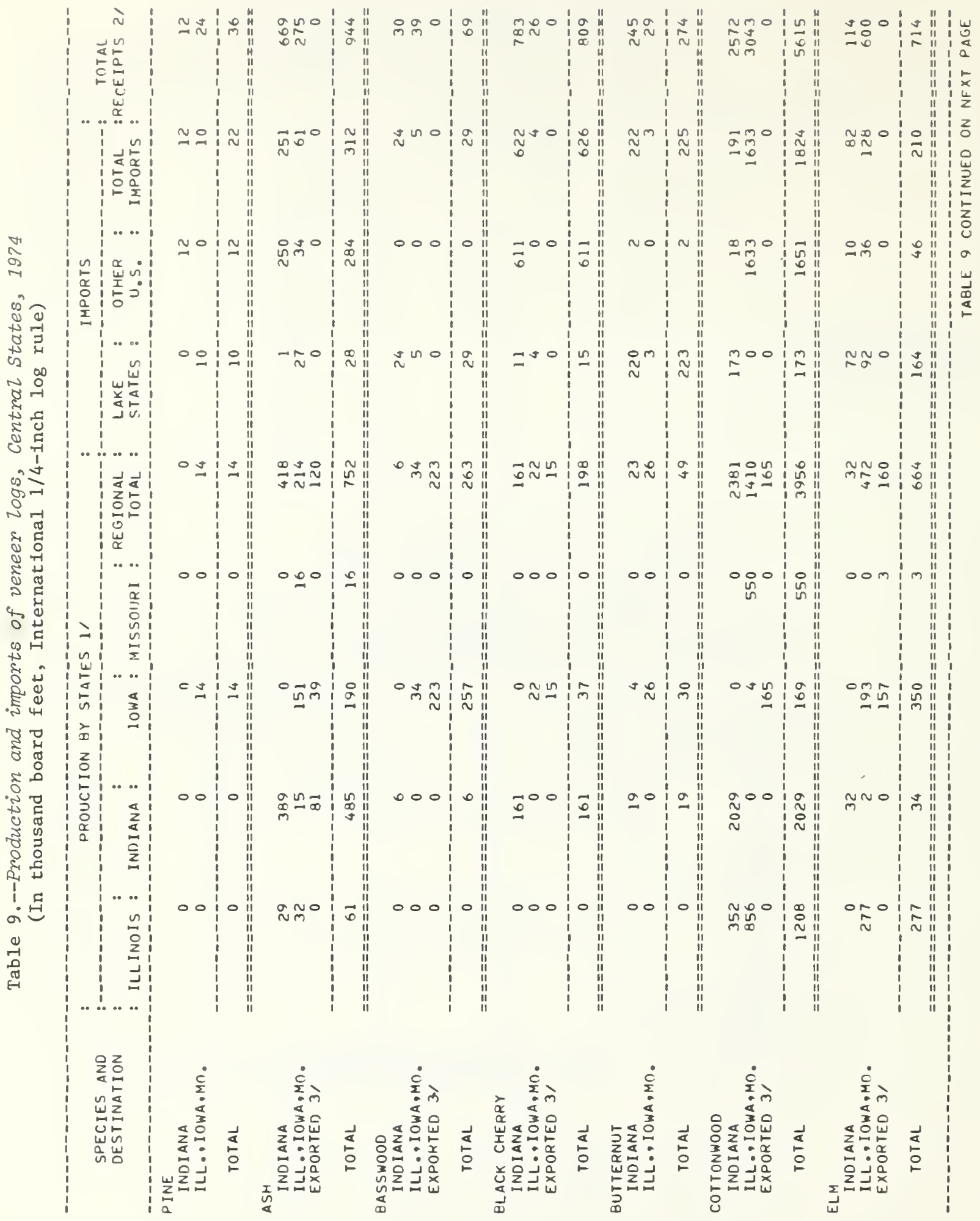




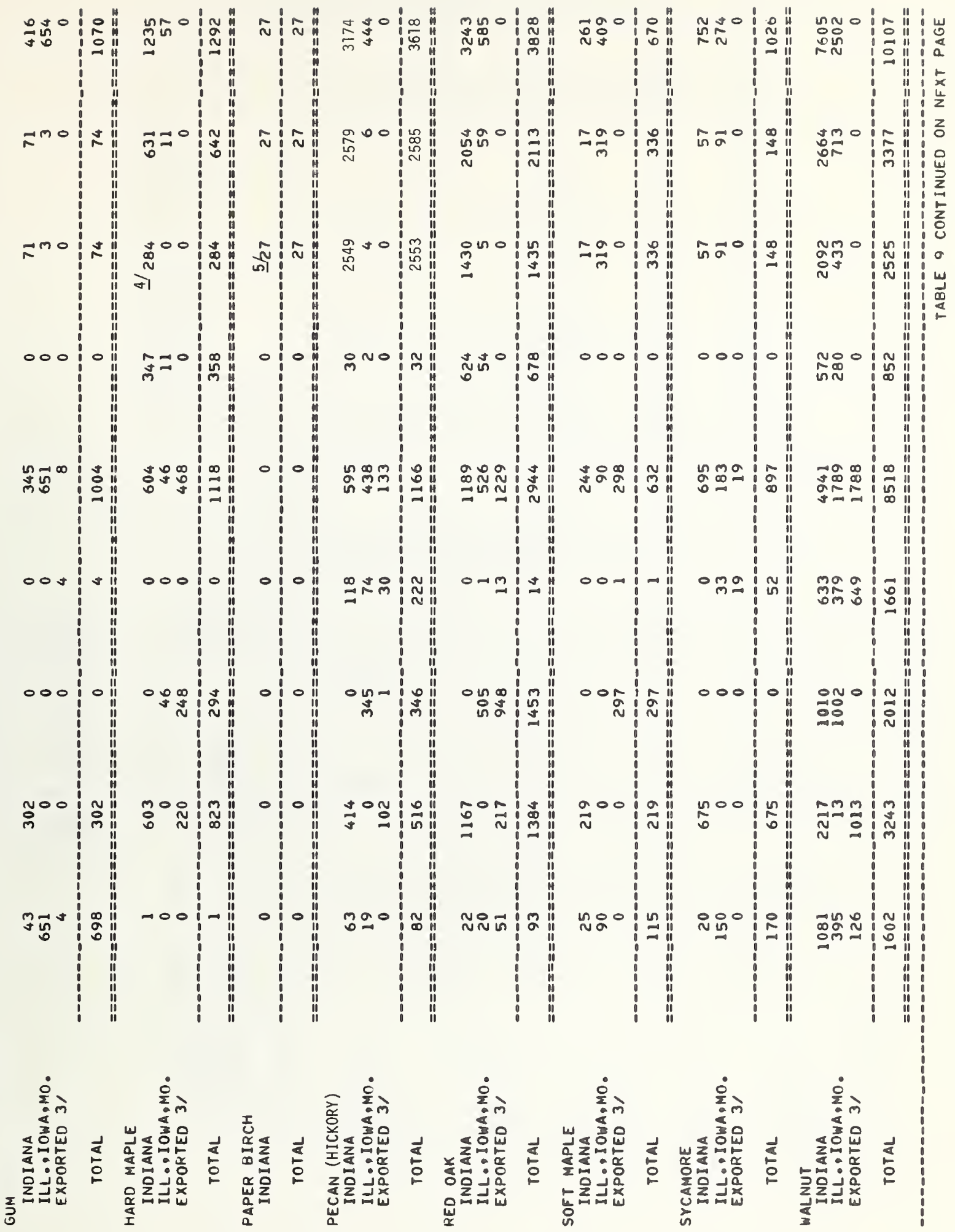




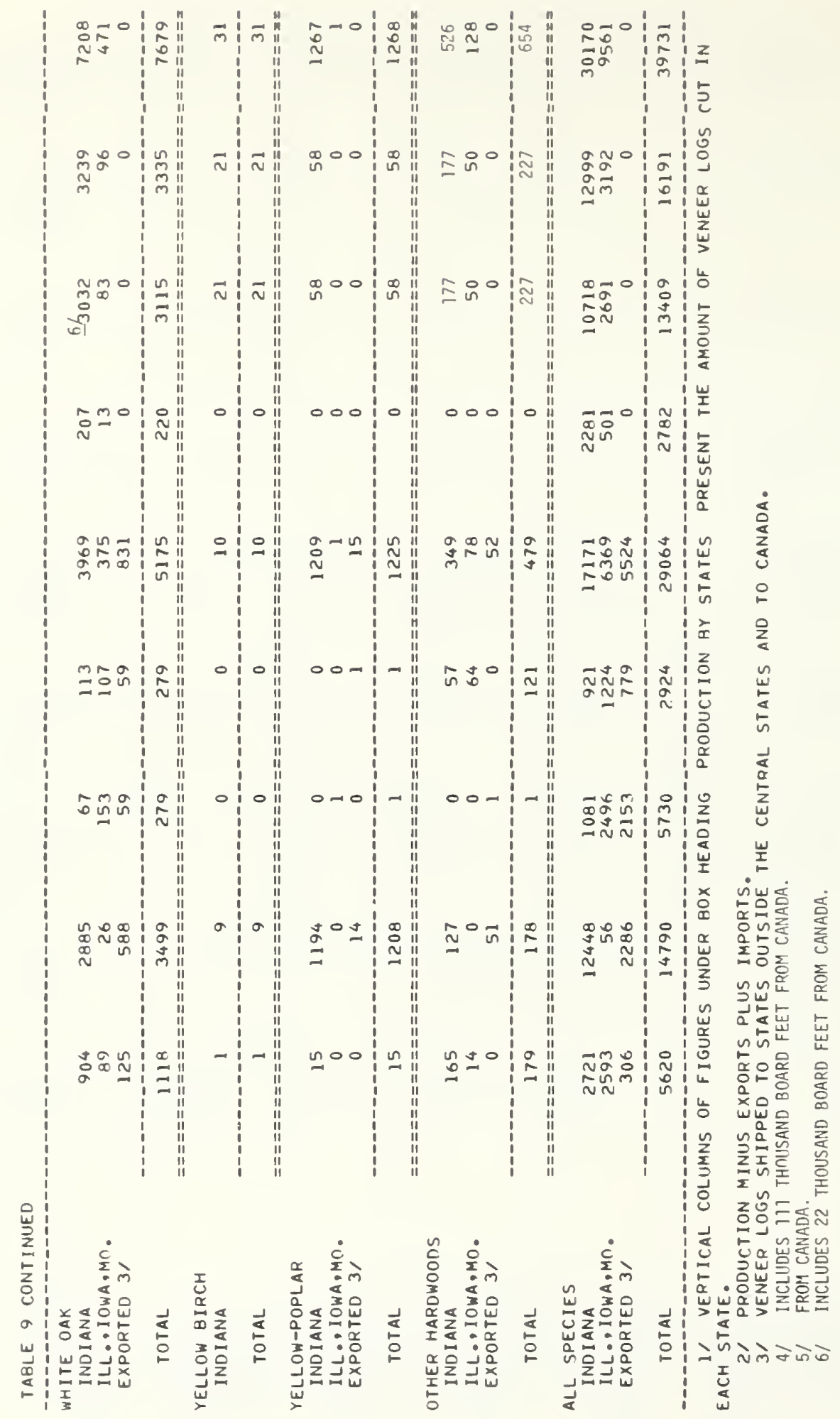


Table 10.--Veneer-log production and receipts in the Central States by species, 1972 and 1974

(In thousand board feet, International 1/4-inch log rule)

\begin{tabular}{|c|c|c|c|c|c|c|}
\hline \multirow{2}{*}{ Spec1es } & \multicolumn{3}{|c|}{ Production } & \multicolumn{3}{|c|}{ Receipts } \\
\hline & 1972 & $: 1974$ & : Change & $: 1972$ & $: 1974$ & : Change \\
\hline Ash & 392 & 752 & +360 & 542 & 944 & +402 \\
\hline Basswood & 263 & 263 & - & 36 & 69 & +33 \\
\hline Black cherry & 86 & 198 & +112 & 855 & 809 & -46 \\
\hline Butternut & 43 & 49 & +6 & 381 & 274 & -107 \\
\hline Cottonwood & 3478 & 3956 & +478 & 4855 & 5615 & +760 \\
\hline Elı & 495 & 664 & +169 & 342 & 714 & +372 \\
\hline Gum & 490 & 1004 & +514 & 606 & 1070 & +464 \\
\hline Hard maple & 1136 & 1118 & -18 & 2425 & 1292 & -1133 \\
\hline Pecan (hickory) & 2398 & 1166 & -1232 & 5690 & 3618 & -2072 \\
\hline Red oak & 1727 & 2944 & +1217 & 1569 & 3828 & +2259 \\
\hline Soft maple & 445 & 632 & +187 & 515 & 670 & +155 \\
\hline Sycamore & 682 & 897 & +215 & 854 & 1026 & +172 \\
\hline Walnut & 10004 & 8518 & -1486 & 10984 & 10107 & -877 \\
\hline White oak & 3314 & 5175 & +1861 & 4034 & 7679 & +3645 \\
\hline Yellow-poplar & 1150 & 1225 & +75 & 1231 & 1268 & +37 \\
\hline Other specles & 109 & 503 & +394 & 137 & 748 & +611 \\
\hline Tota1 & 26212 & 29064 & +2852 & 35056 & 39731 & +4675 \\
\hline
\end{tabular}

Table 11.--Veneer-log production by specres groups for selected. years, Central States, 1956-1974

(In miliion board feet, International 1/4-inch log rule)

\begin{tabular}{|c|c|c|c|c|c|c|c|c|c|c|c|c|}
\hline Year & $\begin{array}{l}: \text { Cotton- } \\
: \text { wood }\end{array}$ & : E1m & $\begin{array}{l}\text { : Hard } \\
\text { : maple }\end{array}$ & $\begin{array}{l}\text { : Pecan } \\
: \text { (h1ckory) }\end{array}$ & $\begin{array}{l}: \text { Red } \\
\text { : oak } \\
\end{array}$ & $\begin{array}{l}: \text { Soft } \\
\text { : maple }\end{array}$ & Sycamore & :Walnut & $\begin{array}{l}\text { : White } \\
\text { : oak }\end{array}$ & $\begin{array}{l}\text { : Yellow- } \\
\text { : poplar }\end{array}$ & $\begin{array}{l}: \text { Other } \\
: \text { spec1es }\end{array}$ & $\begin{array}{cc}: \text { All } \\
: \quad \text { spec1es } \\
\end{array}$ \\
\hline 1956 & 8.0 & 0.9 & 0.8 & 0.1 & 1.1 & 1.8 & 0.6 & 10.6 & 3.4 & 1.6 & 4.2 & 33.1 \\
\hline 1958 & 8.9 & .6 & 1.1 & .1 & 1.0 & .5 & .5 & 10.8 & 2.6 & 1.1 & 5.2 & 32.4 \\
\hline 1960 & 11.2 & 2.0 & 1.2 & .1 & 1.5 & 1.2 & 2.5 & 13.2 & 1.6 & 1.6 & 3.8 & 39.9 \\
\hline 1963 & 7.4 & .7 & 1.6 & .8 & .9 & 1.0 & 1.8 & 15.5 & 1.4 & 2.3 & 4.6 & 38.0 \\
\hline 1966 & 6.2 & $1 / .7$ & 1.4 & 1.1 & 2.3 & .5 & 1.0 & 14.7 & 1.9 & 3.0 & 3.9 & 36.7 \\
\hline 1968 & 4.1 & .7 & 1.3 & 3.0 & 2.1 & .4 & 1.2 & 16.1 & 3.6 & 1.3 & 3.2 & 37.0 \\
\hline 1970 & 4.8 & .6 & .8 & 1.5 & 1.3 & .5 & 1.3 & 8.1 & 1.7 & .8 & 2.1 & 23.5 \\
\hline 1972 & 3.5 & .5 & 1.1 & 2.4 & 1.7 & .4 & .7 & 10.0 & 3.3 & 1.2 & 1.4 & 26.2 \\
\hline 1974 & 4.0 & .7 & 1.1 & 1.2 & 2.9 & .6 & .9 & 8.5 & 5.2 & 1.2 & 2.8 & 29.1 \\
\hline
\end{tabular}

1/ Estimated. 
Table 12.--Veneer-log receipts by species groups for selected years, Central State8, 1956-1974

(In million board feet, International 1/4-inch log rule)

\begin{tabular}{|c|c|c|c|c|c|c|c|c|c|c|c|c|c|c|}
\hline Year & $\begin{array}{l}: \\
:\end{array}$ & $\begin{array}{l}\text { Cotton- } \\
\text { wood }\end{array}$ & Elm & $:$ & $\begin{array}{l}\text { Hard : } \\
\text { maple: ( }\end{array}$ & $\begin{array}{c}\text { Pecan: } \\
\text { h1ckory): }\end{array}$ & $\begin{array}{l}\text { Red } \\
\text { oak } \\
\end{array}$ & $\begin{array}{l}: \text { Soft } \\
: \text { maple }\end{array}$ & : Sycamore: & Walnut: & $\begin{array}{c}\text { h'h1te } \\
\text { oak }\end{array}$ & $\begin{array}{l}\text { : Yellow- } \\
\text { : poplar }\end{array}$ & $\begin{array}{l}: \text { Other } \\
: \text { spec1es }\end{array}$ & $\begin{array}{c}: \text { All } \\
: \quad \text { spec } 1 \text { es } \\
\end{array}$ \\
\hline 1956 & & 10.9 & 0.8 & & 1.2 & 0.6 & 1.3 & 0.8 & 1.3 & 11.4 & 6.7 & 2.8 & 6.6 & 44.4 \\
\hline 1958 & & 10.2 & .6 & & 1.7 & .3 & 1.3 & .3 & 1.2 & 13.0 & 4.8 & 1.9 & 6.3 & 41.6 \\
\hline 1960 & & 10.4 & 1.0 & & 2.9 & .2 & 1.4 & .5 & 2.3 & 17.1 & 3.6 & 2.8 & 8.0 & 50.2 \\
\hline 1963 & & 8.6 & 1.0 & & 3.1 & 1.6 & .9 & .8 & 2.0 & 21.2 & 2.3 & 2.8 & 7.9 & 52.2 \\
\hline 1966 & & 6.7 & $1 / 1.0$ & & 3.6 & 1.6 & 2.0 & .4 & 1.2 & 17.9 & 2.1 & 4.4 & 7.3 & 48.2 \\
\hline 1968 & & 5.7 & -1.0 & & 2.7 & 4.9 & 2.3 & .5 & 1.1 & 18.4 & 5.2 & 1.9 & 5.1 & 48.8 \\
\hline 1970 & & 4.7 & .5 & & 1.3 & 3.0 & .8 & .6 & 1.4 & 10.0 & 2.0 & 1.1 & 2.6 & 28.0 \\
\hline 1972 & & 4.9 & .3 & & 2.4 & 5.7 & 1.6 & .5 & .9 & 11.0 & 4.0 & 1.2 & 2.6 & 35.1 \\
\hline 1974 & & 5.6 & .7 & & 1.3 & 3.6 & 3.8 & .7 & 1.0 & 10.1 & 7.7 & 1.3 & 3.9 & 39.7 \\
\hline
\end{tabular}

I/ Estimated.

Table 13.--Production and disposition of hardwood veneer plant residue by type of residue and use, Central States, 1974

(In thousand cubic feet)

\begin{tabular}{|c|c|c|c|c|}
\hline State and & $:$ & vood res1du & & \\
\hline type of use & Total & $\therefore$ Coarse 1 & Fine 2/ & Bark \\
\hline $\begin{array}{l}\text { Ill1no1s, Iowa and } \\
\text { M1ssour 1 }\end{array}$ & & & & \\
\hline F1ber products & 63.2 & 63.2 & - & - \\
\hline Industrial fuel & 315.4 & 62.9 & 252.5 & 49.8 \\
\hline Domestic fuel & 5.9 & 5.9 & - & 1.6 \\
\hline M1scellaneous 3/ & 28.3 & 28.3 & - & - \\
\hline Not used 41 & 283.7 & 107.0 & 176.7 & 244.1 \\
\hline Total - & 696.5 & 267.3 & 429.2 & 295.5 \\
\hline Ind Ians & & & & \\
\hline F1ber products & 337.5 & 141.4 & 196.1 & - \\
\hline Industrlal fuel & 1446.1 & 395.9 & 1050.2 & 723.3 \\
\hline Domest1c fuel & 33.6 & 18.7 & 14.9 & - \\
\hline M1scellaneous $\underline{3} /$ & 364.7 & 272.9 & 91.8 & 83.0 \\
\hline Not used 41 & 19.8 & 15.5 & 4.3 & 128.6 \\
\hline Total & 2201.7 & 844.4 & 1357.3 & 934.9 \\
\hline All Central State & & & & \\
\hline F1ber products & 400.7 & 204.6 & 196.1 & - \\
\hline Industrial fuel & 1761.5 & 458.8 & 1302.7 & 773.1 \\
\hline Domestic fuel & 39.5 & 24.6 & 14.9 & 1.6 \\
\hline M1scellaneous 3/ & 393.0 & 301.2 & 91.8 & 83.0 \\
\hline Not used $4 /$ & 303.5 & 122.5 & 181.0 & 372.7 \\
\hline Total & 2898.2 & 1111.7 & 1786.5 & 1230.4 \\
\hline
\end{tabular}

1/Sultable for chipping, such as veneer cores.

$\overline{2} /$ Not sultable for chlpping, such as veneer clippings.

$\overline{3} /$ Livestock bedding, mulch, small dimension and specialty 1 tems.

4/ Including residues burned as waste. 
Blyth, James E., and Jerold T. Hahn.

1976. Veneer-log production and receipts, North Central Region, 1974. USDA For. Serv. Resour. Bull. NC-32, 16 p., illus. North Cent. For. Exp. Stn., St. Paul, Minn.

Shows 1974 veneer-log production and receipts by species in the Lake States (Michigan, Minnesota, and Wisconsin) and in the Central States (I1linois, Indiana, Iowa, and Missouri). Comparisons are made with similar data for 1972. Includes tables showing veneer-log production and receipts (for selected years) since 1946 in the Lake States and since 1956 in the Centra1 States.

OXFORD: 832.20:792(77). KEY WORDS: imports, exports, wood residue, bark, veneer mills.

Blyth, James E., and Jerold T. Hahn.

1976. Veneer-log production and receipts, North Central Region, 1974. USDA For. Serv. Resour. Bull. NC-32, 16 p., illus. North Cent. For. Exp. Stn., St. Paul, Minn.

Shows 1974 veneer-log production and receipts by species in the Lake States (Michigan, Minnesota, and Wisconsin) and in the Central States (Illinois, Indiana, Iowa, and Missouri). Comparisons are made with similar data for 1972. Includes tables showing veneer-log production and receipts (for selected years) since 1946 in the Lake States and since 1956 in the Centra1 States.

OXFORD: $\overline{832.20}$ :792(77). KEY WORDS: imports, exports, wood residue, bark, veneer mills. 


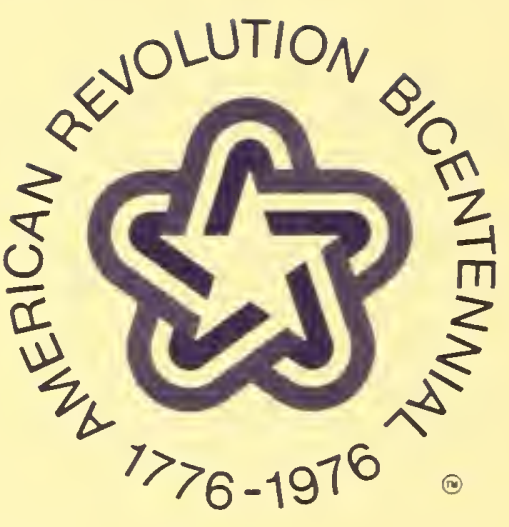

